University of South Carolina

Scholar Commons

Faculty Publications

Physics and Astronomy, Department of

10-6-1986

\title{
Asymmetry in the Magnetoconductance of Metal Wires and Loops
}

\author{
A. D. Benoit \\ S. Washburn \\ C. P. Umbach \\ R. B. Laibowitz \\ Richard A. Webb \\ University of South Carolina - Columbia, webbra@mailbox.sc.edu
}

Follow this and additional works at: https://scholarcommons.sc.edu/phys_facpub

Part of the Physics Commons

\section{Publication Info}

Published in Physical Review Letters, ed. Gene D. Sprouse, Volume 57, Issue 14, 1986, pages 1765-1768.

Benoit, A. D., Washburn, S. Umbach, C. P., Laibowitz, R. B., \& Webb, R. A. (1986). Asymmetry in the magnetoconductance of metal wires and loops. Physical Review Letters, 57(14), 1765-1768. DOI:

10.1103/PhysRevLett.57.1765

(C) Physical Review Letters, 1986, American Physical Society

This Article is brought to you by the Physics and Astronomy, Department of at Scholar Commons. It has been accepted for inclusion in Faculty Publications by an authorized administrator of Scholar Commons. For more information, please contact digres@mailbox.sc.edu. 


\title{
Asymmetry in the Magnetoconductance of Metal Wires and Loops
}

\author{
A. D. Benoit, ${ }^{(a)}$ S. Washburn, C. P. Umbach, R. B. Laibowitz, and R. A. Webb \\ IBM T. J. Watson Research Center, Yorktown Heights, New York 10598
}

(Received 2 June 1986)

\begin{abstract}
Universal conductance fluctuations in wires and Aharonov-Bohm oscillations in loops are not symmetric about $H=0$. The observation of asymmetry in the periodic oscillations is possible when the phase-coherence length of the wave function is comparable to the separation of the voltage probes. In both cases, four-probe measurements yield resistances which depend on lead configuration. The asymmetries appear like Hall voltages, and are consistent with Onsager's relations.
\end{abstract}

PACS numbers: $72.15 . \mathrm{Gd}, 73.60 . \mathrm{Dt}$

Since their observation in experiments, ${ }^{1-3}$ the $h / e$ Aharonov-Bohm oscillations ${ }^{4}$ and the associated universal conductance fluctuations ${ }^{2,3}$ have received a considerable amount of experimental ${ }^{5,6}$ and theoreti$\mathrm{cal}^{7-19}$ attention. Experiments have clearly demonstrated that the transport properties of disordered one-dimensional samples can be probed in the limit where the phase coherence length of the electrons is longer than the size of the sample. In this limit, the electron wave function is coherent across the sample and the averaging over many uncorrelated electron trajectories, which gives rise to both the weak localization and classical behavior, is not operative. Studies of the magnetoconductance of small lines have revealed that it fluctuates randomly as a function of field with an amplitude ${ }^{10,12}$ of $\Delta G \sim e^{2} / h$ and a characteristic field scale given by a few flux quanta ${ }^{10}\left(\Phi_{0}=h / e\right)$ in the area of the sample normal to the field. Measurements on doubly connected devices have shown periodic oscillations in the magnetoresistance with the field scale given by one flux quantum in the average area enclosed by the the loop. ${ }^{1,6}$ One puzzling feature that the experiments have clearly demonstrated is that the conductance $G$ of these small devices is not symmetric $^{1,2}$ upon reversal of the magnetic field, $G(H) \neq G(-H)$. It is the purpose of this Letter to describe and interpret experiments aimed at disclosure of the origin of the asymmetry about $H=0$.
Following the experimental observation of conductance asymmetry which apparently violated fundamental symmetries, ${ }^{20}$ several theoretical papers addressed the question of its origin. The asymmetry has been attributed to magnetic impurities, ${ }^{14}$ Hall-voltage fluctuations, ${ }^{8,18}$ and asymmetry in the conductance of manychannel devices. ${ }^{9}$ In the preceding Letter Büttiker ${ }^{19}$ shows, using the Landauer formulation, ${ }^{21}$ that the asymmetry appears because of the four-probe measuring technique. In contrast, much of the theory has relied upon the formula ${ }^{22} G=e^{2} / h \operatorname{Tr}\left[t t^{\dagger}\right]$, which assumes a two-probe measurement. The importance of four-probe techniques has been noted earlier. ${ }^{4,23}$ By assuming that each lead is connected to an independent thermal reservoir, Büttiker ${ }^{19}$ writes the resistance as a function of the transmission coefficients $\left(T_{m n}\right.$ is the probability for carriers incident in lead $n$ to be transmitted to lead $m$ ),

$$
R_{m n, k l}=\left(h / e^{2}\right)\left(T_{k m} T_{l n}-T_{k n} T_{l m}\right) / D,
$$

where $D$ is a sample-specific constant, independent of lead configuration. The subscripts on the resistance refer to current from lead $m$ to lead $n$ and voltage measured between lead $k$ and $l$. In general, Onsager's relations do not require $R_{m n, k l}(H)=R_{m n, k l}(-H)$. On the basis of the above result, we may write a conductance formula for a ring ${ }^{4,8}$ including the effects of magnetic field on the arms of the ring ${ }^{24}$ as

$$
G=A(H)+\frac{e^{2}}{h}\left[B(H)+C(H) \cos \left(\frac{2 \pi \Phi}{\Phi_{0}}-\beta(H)\right)\right],
$$

where the first term is the average conductance of the sample, and $B(H)$ is the term representing the universal conductance fluctuations. ${ }^{10-12} C(H)$ and $\beta(H)$ represent the random functions which modulate the amplitude and phase of the $h / e$ oscillations, respectively, and $\Phi$ is the flux enclosed by the loop. From Eq. (1), it follows that $\beta(H=0)$ can be any number ${ }^{8,9}$ between $-\pi$ and $\pi$. On the other hand, the use of the function $\operatorname{Tr}\left[t t^{\dagger}\right]$ for the conductance im- plies ${ }^{4}$ that $G$ is symmetric in magnetic field and that $\beta(H=0)$ is restricted to be either 0 or $\pi$.

We have observed asymmetry in all of our magnetoresistance measurements on lone wires and loops. The wires and rings in these experiments have had typical dimensions of $\sim 40 \mathrm{~nm}$ wide and thick and 1.0 $\mu \mathrm{m}$ long or 0.25 to $0.9 \mu \mathrm{m}$ diameter. Devices were manufactured from $99.99+\%$ to $99.9999+\%$ pure po- 


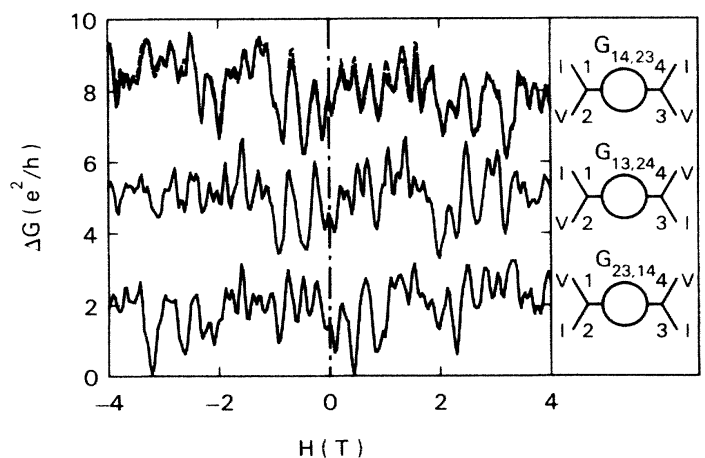

FIG. 1. Magnetoconductance $\Delta G$ measured at $T=0.04 \mathrm{~K}$ for lead configurations $1423[I(1 \rightarrow 4)$ and $V(2 \rightarrow 3)]$, 1324, and 2314 as marked. The dashed line is a different trace for configuration 1423 which indicates the reproducibility in the measurement. The Aharonov-Bohm oscillations ( $h / e$ and $h / 2 e$ ) have been filtered from the data digitally.

lycrystalline gold and silver films having an average grain size of $10-40 \mathrm{~nm}$. The resistances were measured with a lockin amplifier by use of a four-probe technique.

Figure 1 contains three of the many examples of asymmetric traces of the conductance variation in units of $e^{2} / h$ through $H=0$ at $40 \mathrm{mK}$. The sample $(R=55 \Omega$ at $T=4 \mathrm{~K})$ is a 22 -nm-thick, 860 -nm-i.d. polycrystalline $\mathrm{Au}$ ring with a $29-\mathrm{nm}$ linewidth fabricated from $99.9999+\%$ pure $\mathrm{Au}$. The three traces correspond to three different measurement lead arrangements shown schematically in the figure. The $h / e$ Aharonov-Bohm oscillations (0.0069-T period) have been digitally filtered from these traces. The aperiodic conductance fluctuations have rms values of $(0.74,0.70,0.75) e^{2} / h$, respectively (in excellent agreement with theoretical predictions ${ }^{10,12}$ if $L_{\Phi} \geq L$ the sample size). The new result here is that each trace is different and clearly asymmetric about zero field. The dashed line shown on the top trace illustrates the reproducibility which we find upon returning to the first lead configuration after three days of lead-switching experiments. All the individual peak structures are very well reproduced. These fluctuations are random and result from the interaction of the magnetic flux and the conduction states in the wires. ${ }^{7}$ The position of zero magnetic field was determined from criticalfield measurements of a $39-\mu \mathrm{m}$-diam $\mathrm{Al}$ wire doped with $1 \% \mathrm{Si}$ (critical field $-90 \mathrm{Oe}$ ). Even in the absence of a good zero-field marker, it should be apparent that no reasonable shifting of the field axis will make $G(H)=G(-H)$.

It is well known from classical transport theory 25 that any four-wire measurement on an inhomogeneous conductor with the leads placed randomly will in general give six different values for the resistance in the presence of a magnetic field. By adding or subtracting
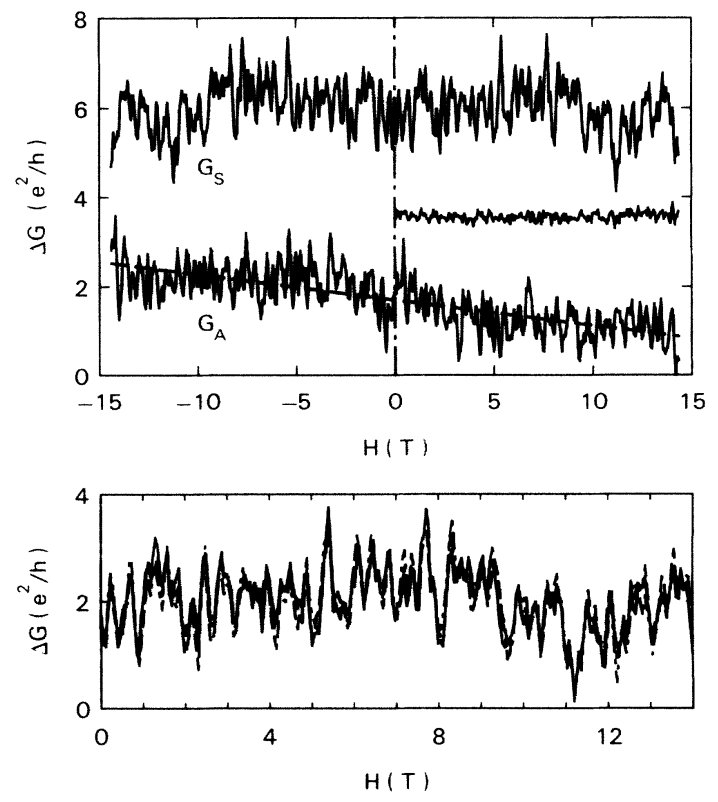

FIG. 2. (a) $G_{\mathrm{S}}=1 / R_{\mathrm{S}}, \quad G_{\mathrm{A}}=1 / R_{\mathrm{A}}$ and $\frac{1}{2}\left[G_{\mathrm{S}}(H)\right.$ $\left.-G_{\mathrm{S}}(-H)\right]$ for the data in Fig. 1. (b) $2 /[R(H)$ $+R(-H)]$ for all lead configurations displayed in Fig. 1 .

the value of the resistance obtained by switching current and voltage leads we obtain three values for the magnetoresistance $R_{\mathrm{S}}$ (symmetric in field) and three values of a Hall-type resistance $R_{\mathrm{A}}$ (antisymmetric). For a long sample (two pairs of leads, well separated), there are two nonzero values for $R_{\mathrm{A}}$ and two for $R_{\mathrm{S}}$. In this case the two values of $R_{\mathrm{S}}$ are equal (and equivalent to $R_{x x}$ for a classical system and symmetric in magnetic field), and the two values of $R_{\mathrm{A}}$ differ. The main point here is that in general any four-terminal measurement of the resistance can contain contributions from Hall effects and $R(H)$ will therefore not be symmetric about zero magnetic field.

In analogy with these classical ideas, we will first assume that the asymmetry apparent in Fig. 1 results from Hall-type effects. Under this assumption, we can use the various lead configurations to construct the equivalent of $R_{\mathrm{S}}$ and $R_{\mathrm{A}}: \quad R_{\mathrm{S}}=\frac{1}{2}\left[R_{14,23}(H)\right.$ $\left.+R_{23,14}(H)\right]$, and $R_{\mathrm{A}}=\frac{1}{2}\left[R_{14,23}(H)-R_{23,14}(H)\right]$. The results of such a decomposition over the full $\pm 15-\mathrm{T}$ range of the data shown in Fig. 1 is displayed in Fig. 2(a). $G_{\mathrm{S}}$ is seen to be almost perfectly symmetric about zero field and $G_{\mathrm{A}}$ is nearly antisymmetric, $G_{\mathrm{A}}(H)=-G_{\mathrm{A}}(-H)$. The interesting feature about the data is that $G_{\mathrm{A}}$ is also a random aperiodic function of magnetic field and that the fluctuations in $G_{\mathrm{S}}$ and $G_{\mathrm{A}}$ are comparable, ${ }^{18} \Delta G_{\mathrm{S}}=0.56 e^{2} / h$ and $\Delta G_{\mathrm{A}}=0.44 e^{2} / h$. (Preliminary measurements of similar fluctuations have been reported earlier. ${ }^{5,8}$ ) The dashed line drawn through the $G_{\mathrm{A}}$ data illustrates the 
weak linear field dependence with a slope of 0.65 $\mu \Omega / \mathrm{Oe}$. Since the sample is not a homogeneous, classical conductor, this value is consistent with the theoretical value $0.34 \mu \Omega / O e$ for a 22 -nm-thick film of gold. The third trace is the residual asymmetry $G_{\mathrm{S}}(H)-G_{\mathrm{S}}(-H)$; the fluctuations in this trace are comparable to the noise in the measurements. Shortrange cross correlation between $G_{12,34}$ and $G_{34,12}$ indicates that at least $90 \%$ of the observed asymmetry can be attributed to $G_{\mathrm{A}}$. The recently predicted finite Hall voltage at zero field ${ }^{13}$ cannot be determined by use of the above procedure. This is a symmetric contribution to the magnetoconductance and cannot be separated from $G_{\mathrm{S}}$ by our decomposition.

We emphasize that the data are very different from expectations based on the classical Hall effect. The simple classical effect would lead to an additive contribution to the conductance which is linear in magnetic field, i.e., it would imply that the amplitude of the asymmetry grows linearly with the magnetic field. In contrast, the average amplitude of the fluctuations in the experiment are roughly constant in magnetic field, and the average amplitude of $G_{\mathrm{A}}$ is also constant in field. This latter observation is consistent with calculations ${ }^{18}$ which suggest that, after growing linearly in field to a value of $\Delta G \sim e^{2} / h$, the average fluctuation amplitude should remain constant.

In Fig. 2(b) we plot $2 /[R(H)+R(-H)]$ for each lead configuration, and all of the curves reproduce $G_{\mathrm{S}}$ from Fig. 2(a). Thus we find the same symmetries as in the classical case; $G_{\mathrm{S}}(H)$ is independent of probe position and is the unique symmetric value representing the conductance of the sample. (Note that this is not generally true for arbitrary sample shape.)

As discussed by Büttiker, ${ }^{19}$ these observations do not violate the Onsager symmetry relations. ${ }^{20}$ Onsager defined a set of coefficients that multiply the chemical potential differences at selected parts of the sample and for any given four-wire lead configuration, the resistance is a nontrivial combination of these coefficients. The symmetry relations defined by Onsager are for these coefficients and in general do not apply to any one value of the measured resistance. When these coefficients are properly calculated for our experiments, the predicted symmetries are precisely those demonstrated in Fig. 2 (a).

Experimentally, the Aharonov-Bohm oscillations also exhibit asymmetry. Figure 3 displays $G_{12,34}$ and $G_{34,12}$ near zero magnetic field for two different rings in a low-field environment. Each trace is an average of three up- and three down-field sweeps, and there is no field hysteresis in the oscillations. The determination of the point $H=0$ was accomplished by measurement of the superconducting transition of a $\mathrm{Zn}$ wire $\left(99.9999+\%\right.$ pure) which marked the fields $H_{C}$ $= \pm 0.0056 \mathrm{~T}$ on sweeps near zero field. From the

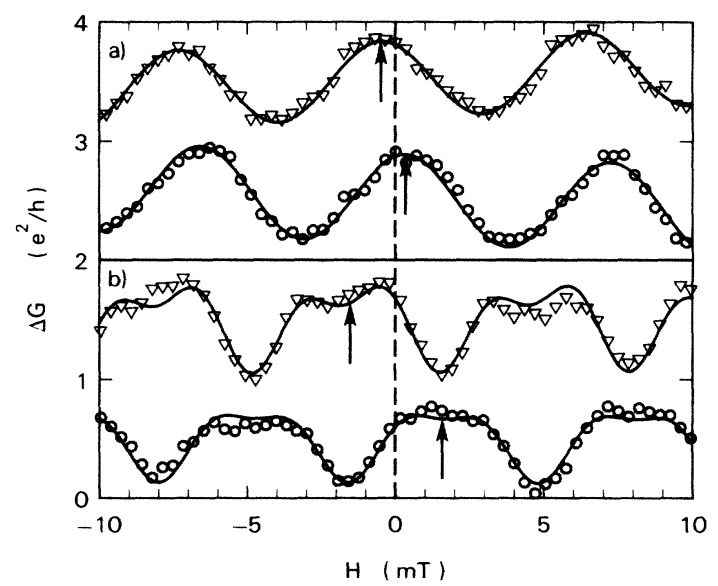

FIG. 3. (a) The asymmetry of the Aharonov-Bohm oscillations for the ring of Fig. 1 for $G_{14,23}$ (circles) and $G_{23,14}$ (inverted triangles). (b) The same measurement for a different ring of similar dimensions. The lines are fitted to the data and the arrows indicate the maximum of the cosine function for the fundamental frequency. These oscillations were studied in a low magnetic field environment.

hysteresis and scatter in this $H_{C}$ measurement we estimate that the error in determining the point $H=0$ is less than $0.1 \mathrm{mT}=0.014 \Phi_{0}$. For the sake of clarity, we have fitted the data with a phase-shifted cosine at the fundamental frequency of the ring (adding a first harmonic for the second sample). To within experimental errors, the phase shift from zero field determined from the fit is clearly nonzero and symmetric upon lead reversal for each ring. The phase shifts are $-25^{\circ}$ and $18^{\circ}$ for the first sample and for the second sample $-86^{\circ}$ and $89^{\circ}$. Several other samples exhibited offsets $\beta$ covering the range $-\pi$ to $\pi$. In all cases the symmetry of the Aharonov-Bohm oscillations with respect to lead configurations was the same as the aperiodic fluctuations as discussed above.

The symmetries displayed in Fig. 3 are precisely those predicted by Büttiker. ${ }^{19}$ In a four-probe measurement, the Aharonov-Bohm oscillations need not be symmetric about zero magnetic field. We emphasize that, in contrast to the effects in Figs. 1 and 2, this asymmetry is not due to the magnetic field piercing the wires, but to an Aharonov-Bohm effect in the proper sense-it is due to the flux threading the loop. The connections to the loop can prevent the observation of an offset if they destroy the wave-functions of the carriers involved, ${ }^{19}$ but they cannot cause the offset.

A possible source of the asymmetry observed in experiments is the presence of magnetic impurities. From recent calculations, ${ }^{14,16}$ it is known that very small changes in the impurity configuration can cause changes in $G$ of order $e^{2} / h$. The flipping of even a few local moments might easily account for the magnitude 
of the asymmetry. This would, however, not explain the difference between various lead configurations. It would cause an asymmetry in $G_{S}$ and would lead to $G_{\mathrm{A}}=0$. This is not what is observed. Furthermore, neither the Aharonov-Bohm oscillations nor the aperiodic fluctuations are hysteretic in magnetic field as expected for magnetic impurities.

To summarize, careful measurements of the magnetoconductance of metallic samples have revealed that both the periodic and the aperiodic components of the conductance are not symmetric upon reversal of the magnetic field. An asymmetric contribution to the aperiodic fluctuations, $\Delta G=0.5 e^{2} / h$, has been observed. We emphasize that this contribution appears like a classical Hall voltage (or transverse conductance) but has a quantum origin. All of the measurements are consistent with Onsager symmetries for four-probe measurements as derived by Büttiker in the preceding Letter.

We acknowledge several enlightening discussions with M. Büttiker and Y. Imry. We are also indebted to H. Fukuyama, P. Lee, M. Ma, and D. Stone for helpful conversations.

(a) Permanent address: Centre National de la Recherche Scientifique, Centre de Recherches sur les très Basses Températures, Grenoble, France.

${ }^{1}$ R. A. Webb, S. Washburn, C. P. Umbach, and R. B. Laibowitz, Phys. Rev. Lett. 54, 2696 (1985); S. Washburn, C. P. Umbach, R. B. Laibowitz, and R. A. Webb, Phys. Rev. B 32, 4789 (1985); C. P. Umbach, C. van Haesendonck, R. B. Laibowitz, S. Washburn, and R. A. Webb, Phys. Rev. Lett. 56, 386 (1986).

${ }^{2}$ R. A. Webb, S. Washburn, C. P. Umbach, and R. B. Laibowitz, in Localization, Interaction, and Transport Phenomena in Impure Metals, edited by G. Bergmann, Y. Bruynseraede, and B. Kramer (Springer-Verlag, Heidelberg, 1985); C. P. Umbach, S. Washburn, R. B. Laibowitz, and R. A. Webb, Phys. Rev. B 30, 4048 (1984).

${ }^{3}$ G. Blonder, Bull. Am. Phys. Soc. 29, 535 (1984); C. P. Umbach, Bull. Am. Phys. Soc. 29, 535 (1984).

${ }^{4}$ M. Büttiker, Y. Imry, R. Landauer, and S. Pinhas, Phys.
Rev. B 31, 6207 (1985), and references cited therein.

${ }^{5}$ W. J. Skocpol et al., in Proceedings of the Thirteenth Yamada Conference on Electronic Properties of Two-Dimensional Systems, Kyoto, Japan, September, 1985 [Surf. Sci. (to be published)].

${ }^{6}$ V. Chandrasekhar, M. J. Rooks, S. Wind, and D. E. Prober, Phys. Rev. Lett. 55, 1610 (1985); S. Datta et al., Phys. Rev. Lett. 55, 2344 (1985).

${ }^{7}$ A. D. Stone, Phys. Rev. Lett. 54, 2692 (1985).

${ }^{8} \mathrm{M}$. Büttiker, in SQUID-85: Superconducting Quantum Interference Devices and Their Applications, edited by D.-A. Halbohm and H. Lübbig (deGruyter, New York, 1985), p. 529; R. A. Webb, S. Washburn, C. P. Umbach, and R. B. Laibowitz, ibid., p. 561.

${ }^{9}$ M. Büttiker and Y. Imry, J. Phys. C 18, L467 (1985).

10P. A. Lee and A. D. Stone, Phys. Rev. Lett. 55, 1622 (1985)

${ }^{11}$ A. D. Stone and Y. Imry, Phys. Rev. Lett. 56, 189 (1986).

12B. L. Al'tshuler, Pis'ma Zh. Eksp. Teor. Fiz. 41, 530 (1985) [JETP Lett. 41, 648 (1985)].

${ }^{13}$ B. L. Al'tshuler and D. E. Khmel'nitskii, Pis'ma Zh. Eksp. Teor. Fiz. 42, 291 (1985) [JETP Lett. 42, 359 (1985)].

${ }^{14}$ B. L. Al'tshuler and B. Z. Spivak, Pis'ma Zh. Eksp. Teor. Fiz. 42, 363 (1985) [JETP Lett. 42, 477 (1985)].

${ }^{15}$ B. L. Al'tshuler and B. I. Shklovskii, to be published.

${ }^{16}$ S. Feng, P. A. Lee, and A. D. Stone, Phys. Rev. Lett. 56, 2772 (1986).

${ }^{17}$ Y. Imry, Europhys. Lett. 1, 249 (1986), and in Perspectives on Condensed Matter Physics, edited by G. Grinstein and E. Mazenko (World Publishing, Singapore, 1986).

${ }^{18} \mathrm{M}$. Ma and P. A. Lee, unpublished.

${ }^{19}$ M. Büttiker, preceding Letter [Phys. Rev. Lett. 57, 1761 (1986)].

${ }^{20}$ L. Onsager, Phys. Rev. 38, 2265 (1931); H. B. G. Casimir, Rev. Mod. Phys. 17, 343 (1945).

${ }^{21}$ R. Landauer, Philos. Mag. 21, 863 (1970).

22P. W. Anderson, D. J. Thouless, E. Abrahams, and D. S. Fisher, Phys. Rev. B 22, 3519 (1980).

${ }^{23}$ H. L. Engquist and P. W. Anderson, Phys. Rev. B 24, 1151 (1981).

${ }^{24}$ A. D. Stone, to be published.

${ }^{25}$ L. D. Landau and E. M. Lifshitz, Electrodynamics of Continuous Media (Pergamon, New York, 1960), p. 92 et seq.; L. J. van der Pauw, Philips Res. Rep. 13, 1 (1958). 